\title{
Paecilomyces tenuipes extract prevents depression-like behaviors in chronic unpredictable mild stress-induced rat model via modulation of neurotransmitters
}

\author{
CHUNGANG LIU ${ }^{1 *}$, JUAN WANG $^{1 *}$, SHIQI XU ${ }^{1}$, SHENGSHU AN ${ }^{1}$, SIYING TANG $^{1}$, JIAN HE $^{2}$, YANG LIU $^{1}$, \\ ROBERT J. LEE ${ }^{1,3}$ and DI WANG ${ }^{1}$ \\ ${ }^{1}$ School of Life Sciences, Jilin University, Changchun, Jilin 130012; ${ }^{2}$ Center for Translational Medicine, Dalian Institute of \\ Chemical Physics, Chinese Academy of Science, Dalian, Liaoning 116023, P.R. China; ${ }^{3}$ Division of Pharmaceutics, \\ College of Pharmacy, The Ohio State University, Columbus, OH 43201, USA
}

Received May 27, 2016; Accepted April 5, 2017

DOI: $10.3892 / \mathrm{mmr} .2017 .6807$

\begin{abstract}
The medicinal fungus Paecilomyces tenuipes exhibits a variety of pharmacological effects, including antidepressive effects. The chronic unpredictable mild stress (CUMS)-induced rat model has served an important role in studies involving antidepressants screening. The aim of the present study was to evaluate the antidepressant-like activity of $P$. tenuipes N45 aqueous extract (PTNE) in a CUMS-induced rat model of behavioral despair depression. Following 4 weeks of PTNE treatment, behavioral tests were conducted to investigate the antidepressant-like activities, and the levels of neurotransmitters and hormones in blood and hypothalamus were measured. The results demonstrated that PTNE treatment significantly increased movement in the forced running test, whereas the immobility time was reduced in the hotplate test and the forced swim test in depression-model rats. PTNE treatment was able to normalize the levels of hormones and neurotransmitters in serum and hypothalamus of CUMS rats. The data demonstrated that PTNE treatment may be a potential pharmaceutical agent in treatment-resistant depression, and the effects of PTNE may be partly mediated through normalizing the levels of neurotransmitters.
\end{abstract}

Correspondence to: Professor Robert J. Lee, Division of Pharmaceutics, College of Pharmacy, The Ohio State University, 500 West 12th Avenue, Columbus, OH 43201, USA

E-mail: lee.1339@osu.edu

Dr Di Wang, School of Life Sciences, Jilin University, 2699 Qianjin Street, Changchun, Jilin 130012, P.R. China

E-mail: jluwangdi@outlook.com

*Contributed equally

Key words: Paecilomyces tenuipes, chronic unpredictable mild stress, antidepression, neurotransmitters

\section{Introduction}

Major depressive disorder (MDD) is a common distress disorder that is characterized by anhedonia, depressed mood and altered cognitive functions (1). According to previous studies, depression will become the leading cause of disability to work and social contact by 2030 (2), which may have serious consequences for individuals and their families (3). Although genetic and environmental factors have been reported to be involved in the etiopathogenesis of depression, the precise mechanisms are still unknown (4). Among the hypotheses regarding the pathogenesis of depression, there is more evidence that implicates hypothalamic-pituitary-adrenal (HPA) axis hyperactivity and neuroendocrine disorders; as well as the involvement of monoamine neurotransmitters $(5,6)$. Current pharmacologic antidepressant therapies target monoamine neurotransmitters in the central nervous system to alleviate depressive symptoms (7). However, the majority of currently available clinical antidepressants have a high risk of adverse effects (8-10), and more than $30 \%$ of patients with depression fail to respond to antidepressant treatment, which not only reflects our incomplete understanding of the etiology of depression, but also indicates a need to discover effective and reliable treatment strategies.

Owing to the safety and effectiveness in alleviating the symptoms of depression, traditional Chinese medicines have been considered as complementary and alternative therapies (11). The well-known medicinal fungus Paecilomyces tenuipes has been used as a crude drug and/or a folk tonic food for antitumor and immunomodulatory therapy (12). The antidepressant-like effects of $P$. tenuipes and its compounds have been reported in animal models (13). One study revealed that $P$. tenuipes treatment regulated the function of the HPA axis in rats, particularly in modulating the serum levels of cortisol and adrenocorticotropic hormone (ATCH) (14). However, the antidepressant-like effects of P.tenuipes and the possible mechanisms of action have rarely been examined.

Based on previous research, the present study aimed to analyze the antidepressant-like effects of $P$. tenuipes in chronic unpredictable mild stress (CUMS)-induced model rats. 
Following 4 weeks of treatment with $P$. tenuipes N45 water extract (PTNE), behavioral tests were conducted to examine its effects on depression-like behaviors in CUMS rats; in addition, hormone and neurotransmitter levels were measured in serum and hypothalamus. The present data may provide experimental evidence to verify whether $P$. tenuipes possesses the potential for use as an adjuvant therapy for depression.

\section{Materials and methods}

PTNE preparation. P. tenuipes N45 (China Center for Type Culture Collection, Wuhan, China) was cultured using a submerged fermentation process on a rotary shaker using a Biostat B fermenter (10 1 flask; 150 rpm; Biostat B; Sartorius $\mathrm{AG}$, Göttingen, Germany) for 5 days at $26^{\circ} \mathrm{C}$. The cultured medium contained glucose $(40 \mathrm{~g} / \mathrm{l})$, peptone $(10 \mathrm{~g} / \mathrm{l})$ and yeast extract powder $(10 \mathrm{~g} / \mathrm{l})$, and was made in water up to 71 . Cultured mycelia were extracted twice in $1,000 \mathrm{ml}$ double distilled water $\left(\mathrm{ddH}_{2} \mathrm{O}\right)$ at $80^{\circ} \mathrm{C}$ for $3 \mathrm{~h}$. Following centrifugation of the water extract at $3,550 \times \mathrm{g}$ and $4^{\circ} \mathrm{C}$ for $10 \mathrm{~min}$, the supernatant was concentrated using an R1002B Rotary Evaporator for $4 \mathrm{~h}$ (pressure, $0.09 \mathrm{mPa}$; temperature, $80^{\circ} \mathrm{C}$; Shanghai Senco Technology Co., Ltd., Shanghai, China) and freeze-dried using a Genesis Pilot Lyophilizer 25ES for further testing. Preliminary determination indicated that PTNE contains $3.9 \%$ polysaccharides, $12.7 \%$ cordycepic acid and $0.3 \%$ adenosine, which were detected via the phenolsulfuricacid method (15) and high performance liquid chromatography (16).

Depression-like rat model establishment and drug treatment procedure. Male Sprague-Dawley rats $(\mathrm{n}=60$; age, 6-weeks-old; weight, 180-220 g) were purchased from the Laboratory Animal Center of Jilin University (Changchun, China) and maintained on a $12 \mathrm{~h}$ light/dark cycle (lights on 07:00-19:00 h) at $23 \pm 1^{\circ} \mathrm{C}$ with water and food available ad libitum. The experimental protocol was approved by The Institution Animal Ethics Committee of Jilin University (Changchun, China).

CUMS rats were established by exposing them to random stressors over a 1 week period. Stressors included: Forced swimming for $5 \mathrm{~min}$ at $4^{\circ} \mathrm{C}, 24 \mathrm{~h}$ wet litter, $12 \mathrm{~h}$ food and water deprivation, $90 \mathrm{sec}$ tail pinch, overnight illumination and $24 \mathrm{~h}$ cage tilt (cages were tilted to $45^{\circ} \mathrm{C}$ from the horizontal). To prevent habituation, all stressors were randomly scheduled every week and repeated for eight weeks; rats were exposed to a different stressor each day of each week, and this was repeated for eight weeks. Rats housed in separated cages that did not receive any treatments served as the control (CTRL) group.

Drug treatments began from the fifth week of CUMS exposure, and were performed $1 \mathrm{~h}$ prior to the daily administration of CUMS. After 8 weeks of exposure to stressors, CUMS-induced depression-like rats were randomly separated into the following 5 groups ( $\mathrm{n}=10 /$ group; all treatments were administered orally for 4 weeks): i) Model group, which received physiological saline $(10 \mathrm{ml} / \mathrm{kg})$; ii) fluoxetine hydrochloride (Flu) group, which received Flu (3 mg/kg; Shanghai Zhongxi Pharmaceutical Co., Ltd., Shanghai, China); and three PTNE treatment groups, which were treated with iii) $0.04 \mathrm{~g} / \mathrm{kg}$, iv) $0.2 \mathrm{~g} / \mathrm{kg}$, or v) $1.0 \mathrm{~g} / \mathrm{kg}$ of PTNE. CTRL rats were treated with $10 \mathrm{ml} / \mathrm{kg}$ of sterile saline $(\mathrm{n}=10)$. The bodyweight of each experimental rat was measured once per week.

\section{Behavioral assessments}

Forced running test (FRT). The FRT was performed as previously described (17). Before formal testing, rats were allowed to run at $20 \mathrm{mph}$ on an FT-200 treadmill (Chengdu Taimeng Science and Technology, Ltd., Chengdu, China) 3 times for $1 \mathrm{~min}$ each. The time until exhaustion was recorded to evaluate the running performance of each rat.

Hotplate test. The hotplate test was performed as reported previously (18). Briefly, rats were placed on a $55 \pm 0.5^{\circ} \mathrm{C}$ surface and paw withdrawal latency time (licking the hind paw of the injured side or jumping at the plate) was measured.

Forced swim test (FST). Rats were placed individually in a Plexiglas cylinder (height, $50 \mathrm{~cm}$; diameter, $20 \mathrm{~cm}$ ) filled with $24 \pm 0.5^{\circ} \mathrm{C}$ water to $40 \pm 1.5 \mathrm{~cm}$. Immobility, which was defined as the lack of motion of the whole body except for the small movements that were necessary to keep the rats head above the water, was recorded during the last $5 \mathrm{~min}$ of the 6 min test.

Hormone and neurotransmitter detection. Upon completion of the aforementioned experiments, blood was collected from the caudal vein, and the rats were sacrificed by injection of pentobarbital $(200 \mathrm{mg} / \mathrm{kg})$. The hypothalamus was collected, weighed and homogenized with $\mathrm{ddH}_{2} \mathrm{O}$. Samples were purified by centrifugation at $4^{\circ} \mathrm{C}$ and $10,000 \mathrm{x} \mathrm{g}$ for $5 \mathrm{~min}$. For detection of serum levels, $1 \mathrm{ml}$ blood was centrifuged at $25^{\circ} \mathrm{C}$ and $2,000 \mathrm{x}$ g for $5 \mathrm{~min}$ and the serum was collected. The levels of 5-hydroxytryptamine (5-HT; cat no. CK-E30326), 5-hydroxyindoleacetic acid (5-HIAA; cat no. CK-E92141R), dopamine (DA; cat no. CK-E30237), 3,4-dihydroxyphenylacetic acid (DOPAC; cat no. CK-E93592R), glucocorticoid receptor (GR; cat no. CK-E30214), norepinephrine (NE; cat no. CK-E30189R), adrenocorticotropic hormone (ACTH; cat no. CK-E30596), acetylcholine (Ach; cat no. CK-E30422) and histamine (His; cat no. CK-E30476) in the hypothalamus and/or serum were measured by enzyme-linked immune sorbent assay (ELISA) kits (Shanghai Yuanye Biotechnology Co., Ltd., Shanghai, China), following the manufacturer's protocol. His and Ach levels were measured in the serum but not in the hypothalamus. Experiments were performed twice independently.

Statistical analysis. All data were expressed as the mean \pm standard error of the mean. Statistical significance was determined by one-way analysis of variance followed by Dunn's test using SPSS software version 16.0 (SPSS Inc., Chicago, IL, USA). $\mathrm{P}<0.05$ was considered to indicate a statistically significant difference.

\section{Results}

Antidepressant-like activities of PTNE. CUMS treatment significantly reduced final bodyweights $(\mathrm{P}<0.001$; Fig. 1A), increased immobility time in FST $(\mathrm{P}<0.001$; Fig. 1B) and the paw withdrawal latency in hotplate test $(\mathrm{P}<0.001$; Fig. $1 \mathrm{C})$, and reduced exhaustive time in FRT $(\mathrm{P}<0.001$; Fig. 1D), compared 
A

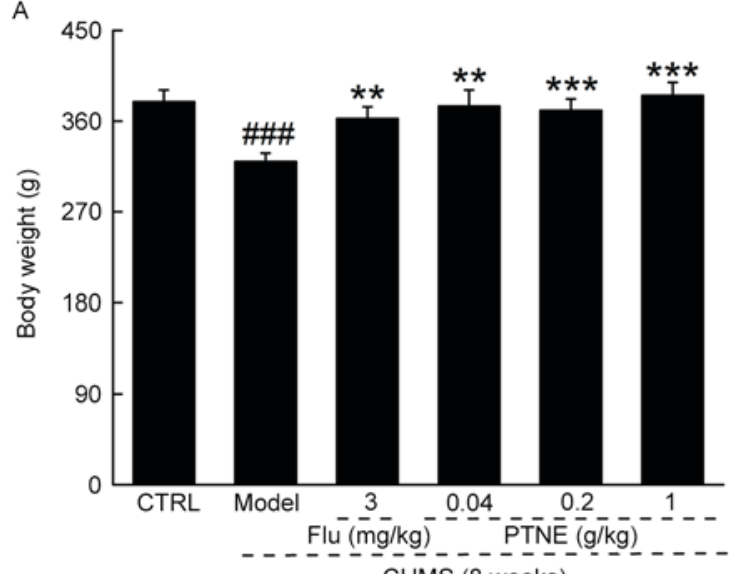
CUMS (8 weeks)

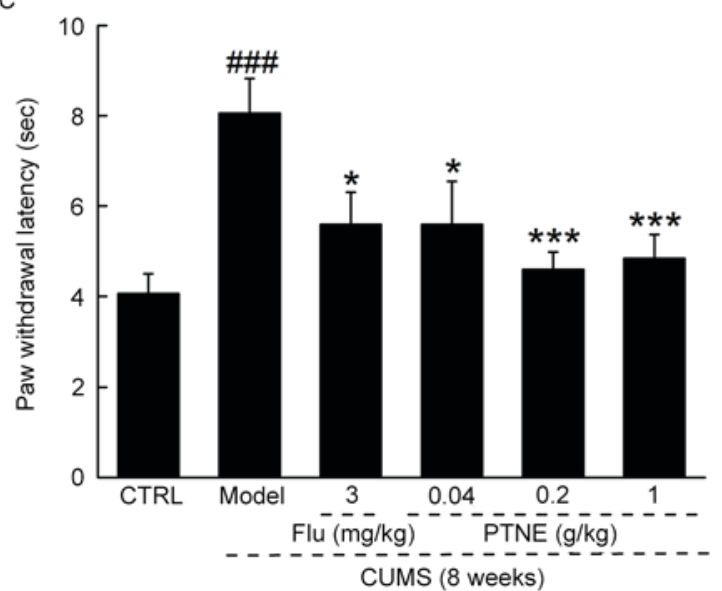

B

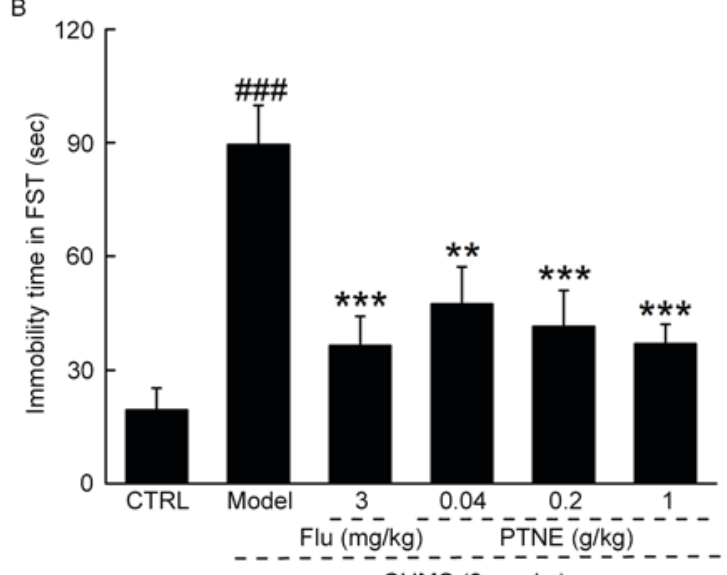

CUMS (8 weeks)

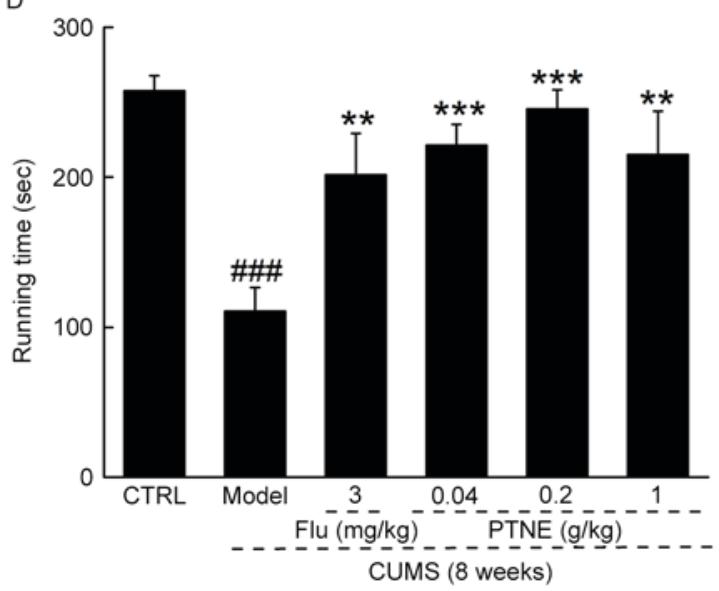

Figure 1. PTNE exhibited anti-depression-like effects in a CUMS-induced rat model. CUMS-induced depression-like rats were treated with PTNE $(0.04,0.2$ and $1.0 \mathrm{~g} / \mathrm{kg})$ and Flu $(3 \mathrm{mg} / \mathrm{kg})$ orally for four weeks. Compared with untreated CUMS model rats, those treated with Flu or the various concentrations of PTNE exhibited (A) higher bodyweight, (B) reduced immobility time in FST, (C) reduced paw withdrawal latency time in the hotplate test, and (D) increased running time in the forced running test compared with untreated CUMS rats. Data are presented as the mean \pm standard deviation of the mean (n=10) and analyzed by a one-way analysis of variance followed by Dunn's test. ${ }^{\# \#} \mathrm{P}<0.001$ vs. CTRL rats; ${ }^{*} \mathrm{P}<0.05,{ }^{* *} \mathrm{P}<0.01$ and ${ }^{* * *} \mathrm{P}<0.001$ vs. untreated CUMS rats. CTRL, control; CUMS, chronic unpredictable mild stress; Flu, fluoxetine hydrochloride; FST, forced swimming test; PTNE, Paecilomyces tenuipes N45 water extract.

with CTRL rats. Compared with untreated CUMS model rats, rats in the PTNE and Flu groups exhibited significantly higher final bodyweights $(\mathrm{P}<0.01$; Fig. $1 \mathrm{~A})$, reduced immobility time in FST $(\mathrm{P}<0.01$; Fig. 1B), suppressed paw withdrawal latency in hotplate test $(\mathrm{P}<0.05$; Fig. $1 \mathrm{C})$ and enhanced exhaustive time in FRT $(\mathrm{P}<0.01$; Fig. 1D), which suggested antidepressant-like activities of PTNE in CUMS-induced depression-like rats.

PTNE regulates neurotransmitter and metabolite levels in serum and hypothalamus. Significant reductions in the serum levels of 5-HT, 5-HIAA, DA, DOPAC, NE, Ach and His were observed in the untreated CUMS model rats compared with CTRL rats $(\mathrm{P}<0.05$; Table I). Flu treatment resulted in a significant increase in the serum concentrations of the neurotransmitters and metabolites in depression-like rats $(\mathrm{P}<0.05$, Table I). CUMS model rats treated with PTNE exhibited similar results as rats treated with Flu: PTNE treatment, particularly at doses of 0.2 and $1.0 \mathrm{~g} / \mathrm{kg}$, increased the serum levels of the neurotransmitters and the metabolites to healthy CTRL-like conditions $(\mathrm{P}<0.05$; Table I). However, PTNE at $0.04 \mathrm{~g} / \mathrm{kg}$ failed to significantly influence the levels of DA, DOPAC, Ach and His, while PTNE at $0.2 \mathrm{~g} / \mathrm{kg}$ failed to significantly reverse the abnormal levels of 5-HIAA and Ach in the serum of CUMS rats (Table I).

Consistent with the effects on serum levels, a significant reduction was observed in the levels of 5-HT, 5-HIAA, DA, DOPAC and NE in the hypothalami of CUMS-induced depression-like rats $(\mathrm{P}<0.05$; Table II), compared with CTRL rats. Certain Flu and PTNE treatments significantly increased the CUMS-inhibited neurotransmitter and metabolite levels in the hypothalamus of depression-like rats $(\mathrm{P}<0.05$; Table II). PTNE at a dose of $1.0 \mathrm{~g} / \mathrm{kg}$ was the only treatment for which a significant increase in the level of hypothalamic DA concentration was identified $(\mathrm{P}<0.01$; Table II).

PTNE regulates hormone levels in serum and hypothalamus. Untreated CUMS model rats exhibited significantly reduced levels of GR and ACTH in both serum and hypothalamus compared with CTRL rats $(\mathrm{P}<0.05$; Fig. 2). Flu and $1.0 \mathrm{~g} / \mathrm{kg}$ PTNE treatments resulted in an increase in the levels of GR and ACTH in the serum compared with untreated CUMS rats (P<0.05; Fig. 2A and C). However, only 0.2 and $1.0 \mathrm{~g} / \mathrm{kg}$ PTNE 
Table I. The effects of Flu and PTNE treatment on neurotransmitter and metabolite levels in the serum of CUMS-induced depression-like rats.

\begin{tabular}{lcccccccc}
\hline Group & Conc. & $\begin{array}{c}5-\mathrm{HT} \\
(\mathrm{ng} / \mathrm{ml})\end{array}$ & $\begin{array}{c}5-\mathrm{HIAA} \\
(\mathrm{ng} / \mathrm{ml})\end{array}$ & $\begin{array}{c}\text { DA } \\
(\mathrm{pg} / \mathrm{ml})\end{array}$ & $\begin{array}{c}\text { DOPAC } \\
(\mathrm{ng} / \mathrm{ml})\end{array}$ & $\begin{array}{c}\text { NE } \\
(\mathrm{ng} / \mathrm{ml})\end{array}$ & $\begin{array}{c}\text { Ach } \\
(\mathrm{pg} / \mathrm{ml})\end{array}$ & $\begin{array}{c}\text { His } \\
(\mathrm{ng} / \mathrm{ml})\end{array}$ \\
\hline CTRL & N/A & $12.2 \pm 1.2^{\mathrm{a}}$ & $4.8 \pm 0.5$ & $122.1 \pm 11.5$ & $29.0 \pm 1.9$ & $8.8 \pm 0.7$ & $628.4 \pm 52.4$ & $39.9 \pm 2.7$ \\
CUMS & N/A & $8.32 \pm 1.1^{\mathrm{a}}$ & $3.3 \pm 0.3^{\mathrm{a}}$ & $89.3 \pm 6.0^{\mathrm{a}}$ & $20.1 \pm 1.9^{\mathrm{a}}$ & $6.9 \pm 0.2^{\mathrm{a}}$ & $461.7 \pm 27.8^{\mathrm{a}}$ & $26.5 \pm 3.3^{\mathrm{a}}$ \\
Flu $(\mathrm{mg} / \mathrm{kg})$ & 3.0 & $13.2 \pm 1.1^{\mathrm{c}}$ & $4.7 \pm 0.4^{\mathrm{b}}$ & $127.3 \pm 14.6^{\mathrm{b}}$ & $27.9 \pm 1.2^{\mathrm{b}}$ & $8.8 \pm 0.4^{\mathrm{c}}$ & $584.7 \pm 38.7^{\mathrm{b}}$ & $34.4 \pm 2.9^{\mathrm{b}}$ \\
PTNE $(\mathrm{g} / \mathrm{kg})$ & 0.04 & $13.8 \pm 1.5^{\mathrm{c}}$ & $4.3 \pm 0.3^{\mathrm{b}}$ & $76.0 \pm 13.3$ & $25.2 \pm 2.2^{2}$ & $8.4 \pm 0.3^{\mathrm{b}}$ & $428.9 \pm 26.9$ & $32.8 \pm 4.9^{2}$ \\
& 0.2 & $11.8 \pm 1.2^{\mathrm{b}}$ & $3.4 \pm 0.2$ & $119.3 \pm 6.2^{\mathrm{b}}$ & $29.7 \pm 3.1^{\mathrm{b}}$ & $8.4 \pm 0.5^{\mathrm{b}}$ & $581.9 \pm 81.9$ & $41.5 \pm 3.2^{\mathrm{b}}$ \\
& 1.0 & $11.3 \pm 1.3$ & $4.2 \pm 0.24^{\mathrm{b}}$ & $121.5 \pm 10.3^{\mathrm{b}}$ & $30.8 \pm 2.4^{\mathrm{b}}$ & $9.9 \pm 1.0^{\mathrm{b}}$ & $680.3 \pm 75.4^{\mathrm{b}}$ & $40.5 \pm 2.6^{\mathrm{b}}$ \\
\hline
\end{tabular}

Data are expressed as the mean \pm standard error of the mean $(n=10)$ and analyzed using a one-way analysis of variance followed by Dunn's test. ${ }^{\mathrm{P}} \mathrm{P}<0.05$ vs. CTRL rats; ${ }^{\mathrm{b}} \mathrm{P}<0.05$ and ${ }^{\mathrm{c}} \mathrm{P}<0.01$ vs. CUMS model rats. 5-HIAA, 5-hydroxyindoleacetic acid; 5-HT, 5-hydroxytryptamine; Ach, acetylcholine; Conc., concentration; CTRL, control; CUMS, chronic unpredictable mild stress; DA, dopamine; DOPAC, 3,4-dihydroxyphenylacetic acid; Flu, fluoxetine hydrochloride; His, histamine; N/A, not applicable; NE, norepinephrine; PTNE, Paecilomyces tenuipes N45 water extract.

Table II. The effects of Flu and PTNE on neurotransmitter and metabolite levels in the hypothalamus of CUMS-induced depression-like rats.

\begin{tabular}{lclcccc}
\hline Group & Conc. & 5-HT (ng/g) & 5-HIAA (ng/g) & DA (pg/g) & DOPAC (ng/g) & NE (ng/g) \\
\hline CTRL & N/A & $183.0 \pm 8.4$ & $35.2 \pm 4.4$ & $2,003.2 \pm 99.9$ & $348.3 \pm 27.2$ & $145.5 \pm 3.0$ \\
CUMS & N/A & $148.2 \pm 12.6^{\mathrm{a}}$ & $23.7 \pm 2.2^{\mathrm{a}}$ & $1,726.4 \pm 61.8^{\mathrm{a}}$ & $255.9 \pm 25.1^{\mathrm{a}}$ & $105.6 \pm 2.9^{\mathrm{b}}$ \\
Flu (mg/kg) & 3 & $187.5 \pm 11.7^{\mathrm{c}}$ & $33.3 \pm 2.9^{\mathrm{c}}$ & $1,710.4 \pm 145.5$ & $323.8 \pm 14.8^{\mathrm{c}}$ & $131.6 \pm 5.0^{\mathrm{e}}$ \\
PTNE $(\mathrm{g} / \mathrm{kg})$ & 0.04 & $190.3 \pm 15.1^{\mathrm{c}}$ & $19.3 \pm 2.0$ & $1,596.1 \pm 180.9$ & $258.1 \pm 22.2$ & $146.6 \pm 4.6^{\mathrm{e}}$ \\
& 0.2 & $203.0 \pm 13.3^{\mathrm{c}}$ & $34.4 \pm 3.7^{\mathrm{c}}$ & $1,944.3 \pm 137.2$ & $350.2 \pm 28.6^{\mathrm{c}}$ & $144.3 \pm 6.1^{\mathrm{e}}$ \\
& 1.0 & $193.1 \pm 7.3^{\mathrm{c}}$ & $32.7 \pm 3.4^{\mathrm{c}}$ & $2,105.0 \pm 97.7^{\mathrm{d}}$ & $315.5 \pm 7.2^{\mathrm{c}}$ & $140.6 \pm 6.2^{\mathrm{e}}$ \\
\hline
\end{tabular}

Data are expressed as the mean \pm standard error of the mean $(n=10)$ and analyzed by a one-way analysis of variance followed by Dunn's test. ${ }^{\mathrm{a}} \mathrm{P}<0.05$ and ${ }^{\mathrm{b}} \mathrm{P}<0.001$ vs. CTRL rats; ${ }^{\mathrm{C}} \mathrm{P}<0.05,{ }^{\mathrm{d}} \mathrm{P}<0.01$ and ${ }^{\mathrm{e}} \mathrm{P}<0.001$ vs. CUMS model rats. 5-HIAA, 5-hydroxyindoleacetic acid; 5-HT, 5-hydroxytryptamine; Conc., concentration; CTRL, control; CUMS, chronic unpredictable mild stress; DA, dopamine; DOPAC, 3,4-dihydroxyphenylacetic acid; Flu, fluoxetine hydrochloride; N/A, not applicable; NE, norepinephrine; PTNE, Paecilomyces tenuipes N45 water extract.

treatment was able to increase GR levels in the hypothalamus $(\mathrm{P}<0.05$; Fig. 2B); whereas neither Flu nor PTNE treatments were able to affect ACTH levels in the hypothalamus in CUMS rats (Fig. 2D).

\section{Discussion}

Depression has been recognized as one of the most prevalent disorders worldwide and results in significant social burden (4). Depression-like behaviors can be successfully produced in rats through environmental manipulations or by genetic and/or pharmacological factors (19). When exposed to CUMS, rats exhibit behavioral disturbances and neurobiological changes that are similar to the symptoms of patients with depression (20); thus, CUMS-induced depression-like model rats have been widely used to evaluate the antidepressive effects of a wide range of drug treatments (21). The present study investigated the antidepressant-like effects of PTNE treatment on CUMS model rats. Exercise promotes physical health, and has been reported to be an efficient antidepressant in patients with depression (22). A previous study associated depression-like phenotypes in rats with mechanical allodynia and transient thermal hyperalgesia in the hotplate test, and suggested that nociceptive responding may be used as an alternative method to assess depression-like behaviors (23). FST is a classical model that is used for measuring behavioral despair and antidepressant response (24). In the present study, PTNE-treated CUMS rats exhibited observable antidepressant effects in FST, hotplate test and forced running test, which led to the exploration of the underlying mechanisms of PTNE on depression-like behavior.

Compared with untreated CUMS rats, PTNE treatment was able to restore the levels of the neurotransmitters in the serum and hypothalamus to a normal standard, particularly the monoamines. In the monoamine hypothesis, depression is connected with the metabolic turnover of DA and 5-HT in the brain (25). 5-HT was reported to regulate various functions within the central nervous system, including impulsivity and mood (26). A recent clinical trial suggested that the concentration of platelet 5-HT1A receptors may serve as a diagnostic 

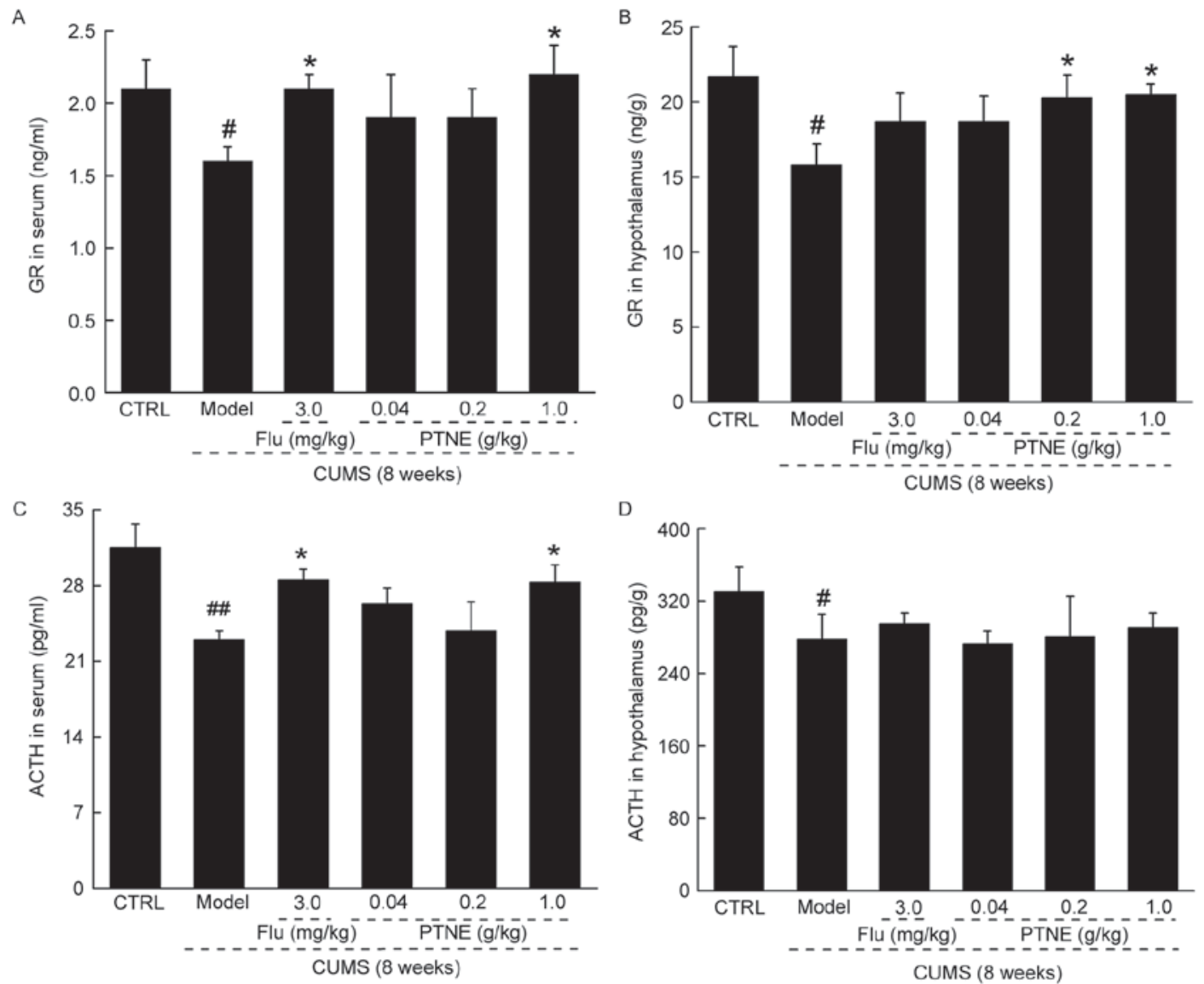

Figure 2. PTNE regulated the levels of ACTH and GR in the serum and hypothalamus of CUMS-induced rats. CUMS-induced depression-like rats were treated with PTNE $(0.04,0.2$ and $1.0 \mathrm{~g} / \mathrm{kg})$ and Flu $(3 \mathrm{mg} / \mathrm{kg}$ ) orally for four weeks. The levels of (A and B) GR and (C and D) ACTH in serum and hypothalamus were analyzed by ELISA analysis. Data are presented as the mean \pm standard deviation of the mean $(n=10)$ and analyzed by a one-way analysis of variance followed by Dunn's test. $\mathrm{P}<0.05$, ${ }^{\# \prime} \mathrm{P}<0.01$ vs. CTRL; ${ }^{*} \mathrm{P}<0.05$ vs. untreated CUMS rats. ACTH, adrenocorticotropic hormone; CTRL, control; CUMS, chronic unpredictable mild stress; Flu, fluoxetine hydrochloride; GR, glucocorticoid receptor; PTNE, Paecilomyces tenuipes N45 water extract.

biomarker for depression (27). Another study reported that a depression-like mouse model was successfully established through the genetic deletion of 5-HT transporter in mice (28). Notably, in the present study, PTNE treatment increased the low levels of 5-HT and 5-HIAA in serum and hypothalamus of depression-like rats. In addition, DA was revealed to be the most abundant catecholaminergic neurotransmitter in the brain, and was implicated in the development of stress-related disorders (29). Consistent with the monoamine hypothesis, a deficit of DA and/or DA metabolites in the brain has been noted in patients with depression (30). Certain antidepressant treatments have been reported to exhibit pharmacological effects related to the modulation of DA functions by increasing the expression of DA receptors or DA levels in the serum and hypothalamus $(31,32)$. In the present study, PTNE treatment not only increased the levels of DA in the serum and hypothalamus of CUMS model rats, but also induced an increase in the levels of NE and DOPAC. NE is another catecholaminergic neurotransmitter that has previously been reported to be involved in mood control (33). Based on the present data, 5-HT and DA may be involved in the antidepressant-like effects of PTNE treatment in CUMS-induced model rats; however, further experimentation is required. The neurotransmitters Ach and His were also examined in the present study, and PTNE treatment was noted to increase their expression levels in CUMS rats. One reported hypothesis suggested that learning difficulties may be explained by the lack of Ach expression in patients with Alzheimer's disease (34). His was demonstrated to act as a modulator in the brain that affects the action of other neurotransmitters (35). Extracellular recordings revealed that His can either excite or depress neuronal activity in different regions of the brain (36). The present study investigated the roles of Ach and His associated with the anti-depressant-like effects of PTNE treatment in CUMS rats, and it was demonstrated that PTNE $(1 \mathrm{~g} / \mathrm{kg})$ treatment significantly increased the levels of Ach and His in the serum of rats compared with CUMS-induced model rats.

Preclinical and clinical studies have revealed that hyperactivity of the HPA axis may be another potential component in the pathophysiology of depression (37). In patients with depression, increased levels of serum ACTH and cortisol indicated an important role for the HPA axis (38). Antidepressants have been reported to ameliorate neurobiological disturbances, including HPA axis hyperactivity, partly by restoring GR function (39). Restraint stress affects the HPA axis and regulates monoamine neurotransmitters in brain (40). Icariin, extracted from Herba Epimedii, displayed antidepressant-like activity in the social defeat mouse model by regulating the function of the HPA axis, particularly by upregulating the levels of GR (41). Similarly, PTNE enhanced the levels of ACTH and GR in serum and hypothalamus of CUMS rats, 
which indicates that its antidepressant-like effect may be associated with the normalization of HPA axis activity. Compared with other synthetic chemical antidepressants, PTNE is an herbal medicine that contains multi-effective components (42), which may target many molecules such as neurotransmitters. The 'systemic targeting' of PTNE, including action in the modulation of neurotransmitters, metabolites and hormones, may relieve depression-like behaviors in a more natural way compared with synthetic antidepressants, so that less adverse side effects would be expected.

In conclusion, the present study demonstrated that PTNE possesses antidepressant-like activities, which may function by regulating the levels of neurotransmitters, metabolites and hormones. The data provided experimental evidence in support of the potential use of PTNE against major depression.

\section{Acknowledgements}

The present study was supported by the 'Twelfth Five-Year' Science and Technology Planning Project of Jilin Province in China (grant no. 2014B033), and The Science and Technology Development Program of Jilin Province in China (grant no. 20160520036JH).

\section{References}

1. Kessler RC: The costs of depression. Psychiat Clin N Am 35: 1, 2012.

2. Mathers CD and Loncar D: Projections of global mortality and burden of disease from 2002 to 2030. PLoS Med 3: e442, 2006.

3. Luppa M, Heinrich S, Angermeyer MC, König HH and Riedel-Heller SG: Cost-of-illness studies of depression: A systematic review. J Affect Disord 98: 29-43, 2007.

4. Krishnan V and Nestler EJ: The molecular neurobiology of depression. Nature 455: 894-902, 2008.

5. Li X, Li L, Shen LL, Qian Y, Cao YX and Zhu DN: Changes of adrenomedullin and its receptor components mRNAs expression in the brain stem and hypothalamus-pituitary-adrenal axis of stress-induced hypertensive rats. Sheng Li Xue Bao 56: 723-729, 2004.

6. Sartori SB, Whittle N, Hetzenauer A and Singewald N: Magnesium deficiency induces anxiety and HPA axis dysregulation: Modulation by therapeutic drug treatment. Neuropharmacology 62: 304-312, 2012.

7. Zhou XJ, Liu M, Yan JJ, Cao Y and Liu P: Antidepressant-like effect of the extracted of Kai Xin San, a traditional Chinese herbal prescription, is explained by modulation of the central monoaminergic neurotransmitter system in mouse. J Ethnopharmacol 139: 422-428, 2012.

8. Ma Z, Ji W, Qu R, Wang M, Yang W, Zhan Z, Fu Q and Ma S: Metabonomic study on the antidepressant-like effects of banxia houpu decoction and its action mechanism. Evid Based Complement Alternat Med 2013: 213739, 2013

9. Kang A, Hao H, Zheng X, Liang Y, Xie Y, Xie T, Dai C, Zhao Q, Wu X, Xie L and Wang G: Peripheral anti-inflammatory effects explain the ginsenosides paradox between poor brain distribution and anti-depression efficacy. J Neuroinflammation 8: 100, 2011.

10. Cui M, Li Q, Zhang M, Zhao YJ, Huang F and Chen YJ: Long-term curcumin treatment antagonizes masseter muscle alterations induced by chronic unpredictable mild stress in rats. Arch Oral Biol 59: 258-267, 2014.

11. Thachil AF, Mohan R and Bhugra D: The evidence base of complementary and alternative therapies in depression. J Affect Disorders 97: 23-35, 2007.

12. Lee DH, Park T and Kim HW: Induction of apoptosis by disturbing mitochondrial-membrane potential and cleaving PARP in Jurkat $T$ cells through treatment with acetoxyscirpenol mycotoxins. Biol Pharm Bull 29: 648-654, 2006.

13. Kan H, Ming L, Li C, Kan H, Sun B and Liang Y: Antidepressant effect of bioactive compounds from Paecilomyces tenuipes in mice and rats. Neural Regen Res 5: 1568-1572, 2010.
14. Yin YY, Ming L, Zheng LF, Kan HW, Li CR and Li WP: Bioactive compounds from Paecilomyces tenuipes regulating the function of the hypothalamo-hypophyseal system axis in chronic unpredictable stress rats. Chin Med J (Engl) 120: 1088-1092, 2007.

15. Jiang Y, Qi X, Gao K, Liu W, Li N, Cheng N, Ding G, Huang W, Wang Z and Xiao W: Relationship between molecular weight, monosaccharide composition and immunobiologic activity of Astragalus polysaccharides. Glycoconj J 33: 755-761, 2016.

16. Li SP, Yang FQ and Tsim KW: Quality control of Cordyceps sinensis, a valued traditional Chinese medicine. J Pharm Biomed Anal 41: 1571-1584, 2006.

17. Nakagawasai O, Yamada K, Nemoto W, Fukahori M, Tadano T and Tan-No K: Liver hydrolysate assists in the recovery from physical fatigue in a mouse model. J Pharmacol Sci 123: 328-335, 2013.

18. Espejo EF and Mir D: Structure of the rat's behaviour in the hot plate test. Behav Brain Res 56: 171-176, 1993.

19. Overstreet DH and Steiner M: Genetic and environmental models of stress-induced depression in rats. Stress Med 14: 261-268, 1998.

20. Ji WW, Li RP, Li M, Wang SY, Zhang X, Niu XX, Li W, Yan L, Wang Y, Fu Q and Ma SP: Antidepressant-like effect of essential oil of Perilla frutescens in a chronic, unpredictable, mild stress-induced depression model mice. Chin J Nat Med 12: 753-759, 2014.

21. Yan WJ, Tan YC, Xu JC, Tang XP, Zhang C, Zhang PB and Ren ZQ: Protective effects of silibinin and its possible mechanism of action in mice exposed to chronic unpredictable mild stress. Biomol Ther (Seoul) 23: 245-250, 2015.

22. Bjørnebekk A, Mathé AA and Brené S: Running has differential effects on NPY, opiates, and cell proliferation in an animal model of depression and controls. Neuropsychopharmacology 31: 256-264, 2006.

23. Burke NN, Hayes E, Calpin P, Kerr DM, Moriarty O, Finn DP and Roche M: Enhanced nociceptive responding in two rat models of depression is associated with alterations in monoamine levels in discrete brain regions. Neuroscience 171: 1300-1313, 2010.

24. Detke MJ, Rickels M and Lucki I: Active behaviors in the rat forced swimming test differentially produced by serotonergic and noradrenergic antidepressants. Psychopharmacology (Berl) 121: 66-72, 1995.

25. Steru L, Chermat R, Thierry B and Simon P: The tail suspension test: A new method for screening antidepressants in mice. Psychopharmacology (Berl) 85: 367-370, 1985.

26. Mohammad-Zadeh LF, Moses L and Gwaltney-Brant SM: Serotonin: A review. J Vet Pharmacol Ther 31: 187-199, 2008.

27. Zhang ZJ, Wang D, Man SC, Ng R, McAlonan GM, Wong HK, Wong W, Lee J and Tan QR: Platelet 5-HT(1A) receptor correlates with major depressive disorder in drug-free patients. Prog Neuropsychopharmacol Biol Psychiatry 53: 74-79, 2014.

28. Gobbi G, Murphy DL, Lesch KP and Blier P: Modifications of the serotonergic system in mice lacking serotonin transporters: An in vivo electrophysiological study. J Pharmacol Exp Ther 296: 987-995, 2001

29. Chrousos GP: Stress and disorders of the stress system. Nat Rev Endocrinol 5: 374-381, 2009.

30. Lambert G, Johansson M, Agren H and Friberg P: Reduced brain norepinephrine and dopamine release in treatment-refractory depressive illness: Evidence in support of the catecholamine hypothesis of mood disorders. Arch Gen Psychiatry 57: 787-793, 2000.

31. Di Giovanni G, Svob Strac D, Sole M, Unzeta M, Tipton KF, Mück-Šeler D, Bolea I, Della Corte L, Nikolac Perkovic M, Pivac N, et al: Monoaminergic and histaminergic strategies and treatments in brain diseases. Front Neurosci 10: 541, 2016.

32. Ainsworth K, Smith SE, Zetterström TS, Pei Q, Franklin M and Sharp T: Effect of antidepressant drugs on dopamine D1 and D2 receptor expression and dopamine release in the nucleus accumbens of the rat. Psychopharmacology (Berl) 140: 470-477, 1998.

33. Pirke KM: Central and peripheral noradrenalin regulation in eating disorders. Psychiat Res 62: 43-49, 1996.

34. Gareri P, Castagna A, Cotroneo AM, Putignano D, Conforti R, Santamaria F, Marino S and Putignano S: The Citicholinage Study: Citicoline plus cholinesterase inhibitors in aged patients affected with Alzheimer's disease study. J Alzheimers Dis 56: 557-565, 2017.

35. Brown RE, Stevens DR and Haas HL: The physiology of brain histamine. Prog Neurobiol 63: 637-672, 2001.

36. Geller HM, Springfield SA and Tiberio AR: Electrophysiological actions of histamine. Can J Physiol Pharmacol 62: 715-719, 1984. 
37. Deuschle M, Bode L, Schnitzler P, Meyding-Lamadé U, Plesch A, Ludwig H, Hamann B and Heuser I: Hypothalamic-pituitary-adrenal (HPA) system activity in depression and infection with Borna disease virus and Chlamydia pneumoniae. Mol Psychiatry 8: 469-470, 2003.

38. Varghese FP and Brown ES: The hypothalamic-pituitary-adrenal axis in major depressive disorder: A brief primer for primary care physicians. Prim Care Companion J Clin Psychiatry 3: $151-155,2001$

39. Mason BL and Pariante CM: The effects of antidepressants on the hypothalamic-pituitary-adrenal axis. Drug News Perspect 19: 603-608, 2006.

40. Bao L, Yao XS, Zhao L, Lu YQ and Kurihara H: Correlation between changes of central neurotransmitter expression and stress response in mice-A restraint time-course analysis. Neural Regeneration Res 3: 167-171, 2008.
41. Wu J, Du J, Xu C, Le J, Xu Y, Liu B and Dong J: Icariin attenuates social defeat-induced down-regulation of glucocorticoid receptor in mice. Pharmacol Biochem Behav 98: 273-278, 2011.

42. Song J, Xing G, Cao J, Teng L, Li C, Meng Q, Lu J, Zhou Y, Liu Y, Wang D and Teng L: Investigation of the antidepressant effects of exopolysaccharides obtained from Marasmius androsaceus fermentation in a mouse model. Mol Med Rep 13: 939-946, 2016. 